Japanese Psychological Research

1986, Vol. 23, No.2, $77-86$

\title{
The effect of stimulus organization on numerosity discrimination
}

\author{
TADASU OYAMA ${ }^{1}$ \\ Department of Psychology, Faculty of Letters, Lniversity of Tokyo, Bunkyo-ku, Tokyo 113
}

The effect of stimulus organization on numerosity discrimination was studied, where 24, 40,4 , and 13 undergraduate students served as the subjects in four experiments. A test pattern consisting of 1 to 15 dots was presented for 20 to $200 \mathrm{~ms}$. The subject's task was to report the perceived number of dots as soon as possible. The results of Experiment 1 indicated that the numerosity discrimination was better for the stimulus patterns consisting of four or five spatially separated subgroups of dots, than for the patterns consisting of dots distributed in the whole area with an equal probability. However, Experiments 2 and 3 indicated that this improved numerosity discrimination only occurs when dot-patterns in subgroups are simple and regular. Experiment 4 showed that the improved numerosity discrimination is correlated with shorter reaction times, and suggested that the effect of stimulus organization can be attributed to more rapid processing, by means of a strategy in which stimulus-dots in each group is subitized and then summed up together. Perceptual grouping is not necessarily accompanied by improved numerosity discrimination.

Key words: numerosity discrimination, stimulus organization, simplicity, regularity, perceptual grouping, span of attention.

As quoted by Jevons (1871), the first systematic investigator on numerosity discrimination, Sir William Hamilton (1859) claimed "If you throw a handful of marbles on the floor, you will find it difficult to view at once more than six, or seven at most, without confusion; but if you group them into twos, or threes, or fives, you can comprehend as many groups as you can units, because the mind considers these groups only as units...." This effect of grouping can be called

I Experiments 1,2, and 3 were conducted when the author was at Chiba University. The author is indebted to Takao Miyamoto, Machiko Kuwabara, Mitsuhiro Matsumoto, Atsuko Takagi, and Takeo Watanabe for their assistance in running experiments, and to Kazunori Morikawa and Shinichi Kita for their assistance in analyzing data. Thanks are also due to Hiroshi Ono, York University, for his suggestion on the experimental design of Experiment 2 and to Yuko Kimura for her suggestions on English expression. Preliminary reports were given at the 40th, 43rd, and 45th Annual Conventions of the Japanese Psychological Association, 1976, 1979 and 1981. "chunking" from the modern information processing view in psychology (Miller, 1956). It also suggests that stimulus organization, which frequently induces perceptual grouping, may also improve numerosity discrimination. Perceptual grouping is greatly determined by stimulus parameters, such as proximity, similarity, closure, good Gestalt, etc., as pointed out by Wertheimer (1923).

Recently many experimental studies have been carried out on various aspects on numerosity discrimination (Atkinson, Campbell, \& Francis, 1976; Oyama, Kikuchi, \& Ichihara, 1981; Woodworth \& Schlosberg, 1954), but only a few have been conducted on relation of grouping to numerosity discrimination (Atkinson, Francis, \& Campbell, 1976; Fujii, 1967). In the present study, an attempt was made to examine systematically the effect of some stimulus parameters, which are known as factors of perceptual grouping, on numerosity discrimination. 


\section{Experiment 1}

This cxperiment was a pilot study to investigate the effect of spatial arrangement, which divides stimulus dots into spatially separated subgroups, on numerosity discrimination. Here, a "subgroup" means a set of stimulus dots, within which inter-dots distances are relatively smaller than those between dots of different subgroups, irrespective of their perceived relations.

\section{Method}

The experiment was conducted in a small seminar room whose windows were covered by blinds. It was a group experiment with a total of 24 subjects (undergraduate students), who were divided into two groups of 11 and 13 subjects. Each group participated in either of the two sessions. The subjects were seated at the distances of 2.80 to $4.90 \mathrm{~m}$ from the screen, on lines intersecting its surface at angles of 60 to 120 degrees. The subjects all had normal or corrected-to-normal visual acuity.

A hundred and seventy-four stimulus slides were divided into three sets, A, B, and $\mathrm{G}$, with 58 slides in each set. Each of the slides contained 4 to 14 black dots. Six different patterns were used for each of nine numerosities of dots, 4 to 12 , and two different patterns for 13 and 14 dots. The stimulus dots in set A were random samples from the points of intersections in the whole $8 \times 8$ matrix shown in Fig. $1 \mathrm{~A}$. Those in Set B were random samples from the 20 dots as shown by vertices of the five squares in Fig. $1 \mathrm{~B}$. Those in Set $\mathrm{G}$ were random samples from 16 dots as shown by vertices of the four squares in Fig. $1 \mathrm{C}$. The dots were distributed with an equal probability over the whole area in Set A, while they were placed in four or five separate areas in Sets B and C. The dots in Sets B and $\mathrm{C}$ were easily seen in separate subgroups due to the factors of proximity, simplicity and regularity (good Gestalt), as discussed later, as shown by some examples of stimulus patterns in Fig. 2.

The dots projected on the screen were $3.5 \mathrm{~cm}$ in diameter and the center-tocenter distance between the two nearest dots was $7.0 \mathrm{~cm}$ on the screen. All the dots were distributed in a $53 \times 53 \mathrm{~cm}$ area, which subtended a visual angle of 11 degrees for the nearest subject and 6 degrees for the furthest subject. The luminance of the white background on the screen was $50 \mathrm{~cd} / \mathrm{m}^{2}$, and the illuminance on the subject's desk was 20 to $200 \mathrm{~lx}$.

All of the three sets of slides were mixed up and presented, using a slide projector with a Ralph Gerbrands electronic tachistoscopic shutter for $200 \mathrm{~ms}$ each in random order, with an interval of $12 \mathrm{~s}$, after a practice sessions of 10 slides containing different patterns of the same type. Each stimulus slide was presented approximately $1 \mathrm{~s}$ after the experimenter's verbal "Ready" signal. The subject was asked to fixate the center of the screen when the "Ready" signal was given, and to write down the perceived number of dots in

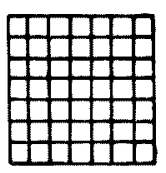

A

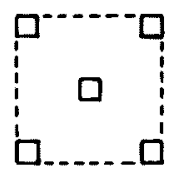

B

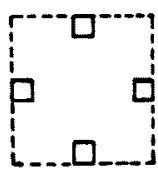

C
Fig, 1. The matrices from whose vertices and points of intersection the positions of stimulus dots were sampled in the Stimulus Sets A, B, and C of Experiment 1 .

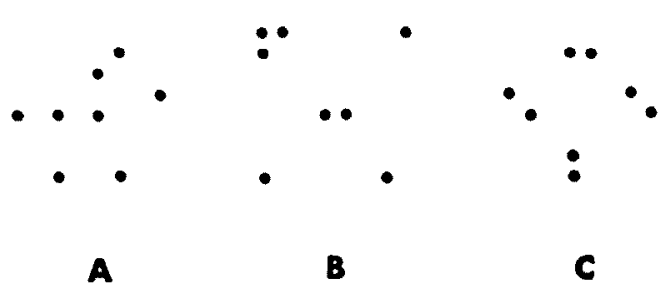

Fig. 2. Examples of the stimulus patterns used in the Sets $A, B$, and $C$ of Experiment 1 . 
a blank table on a sheet of paper after the exposure of each stimulus.

\section{Results and Discussion}

Figure 3 showed the percentage of correct responses of all the subjects as a function of the presented number of dots. The solid, broken, and dotted lines represent the results of Sets $A, B$, and $C$, respectively. The results of Sets $B$ and $\mathrm{C}$ were different from the results of Set A. The percentage of correct responses was markedly higher in Sets B and C than in Set A, especially when the number of dots was greater than 7. Chi-square tests revealed that the percentage of correct responses was greater in Set B than in Set A for stimuli with 8 to 12 dots $\left(\chi^{2}=21.50\right.$, $15.76,13.58,17.01$, and 11.02, $p<0.01$ ), and greater in Set $C$ than in Set $A$ for stimuli with 8 to 11 dots $\left(\chi^{2}=22.80,9.61\right.$, 7.39 , and 8.85, $p<0.01$ ).

If we define the "span of attention", according to the tradition (Hunter \& Sigler, 1940; Woodworth \& Schlosberg, 1954), as the abscissa value where a curve of percentage of correct responses crosses the $50 \%$ level, the span of attention obtained by linear interpolation was 8.8 in Set A, 13.1 in Set B, 11.3 in Set C. The spans of attention in Sets $B$ and $C$ were definitely larger than that in Set A.

The results of chi-square tests and the obtained "span of attention" values

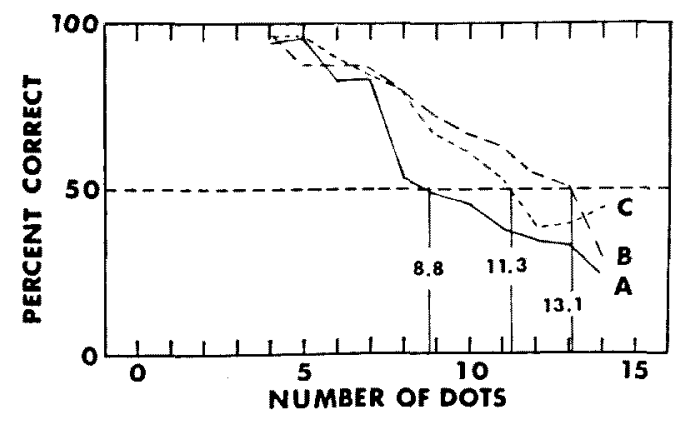

Fig. 3. Percentage of correct responses as a function of the number of dots for the Sets $A, B$, and $\mathrm{C}$ of Experiment 1. clearly indicated that the numbers of dots in Sets $B$ and $C$ were more easily perceived than in Set $\mathrm{A}$. However, these facts do not directly imply that the spatial separation of subgroups always improves numerosity discrimination. The method of pattern production used in Sets B and C produced not only spatially separated subgroups of dots, but also simple and regular patterns in these subgroups. The varieties of patterns in subgroups in Sets $B$ and $C$ were very limited: points (unity), vertical, horizontal, 45 degrees oblique, or 135 degrees oblique straight lines (pairs), right-angled triangles (triples), and squares (quadruples). Hence the simplicity and regularity of patterns in subgroups of dots may be a factor for improving numerosity discrimination. The effect of this factor will be examined in the next experiment.

The exposure duration used in this experiment was $200 \mathrm{~ms}$. This is probably a little longer than the time required for a glance. Eye movements can occur in $200 \mathrm{~ms}$. The effect of exposure duration will also be examined in the next experiment.

\section{Experiment 2}

This experiment was designed firstly find out whether the advantage of Sets $\mathrm{B}$ and $\mathrm{C}$ over Set $\mathrm{A}$ in Experiment 1 was caused by spatial separation of subgroups or by simplicity and regularity of patterns in subgroups and secondly to examine the effect of exposure duration.

\section{Method}

The condition and procedure were similar to those in Experiment 1. Forty undergraduate students served in either of two sessions, 20 for each session. They all had normal or corrected-tonormal visual acuity. They were seated at distances of 2.2 to $5.0 \mathrm{~m}$ from the screen, on lines intersecting its surface at angles of 55 to 125 degrees. 
$\Lambda$ total of 104 stimulus slides, consisting of four sets, were presented in random order. Half the slides in each set were exposed for $200 \mathrm{~ms}$, as in Experiment 1, and the other half for $50 \mathrm{~ms}$. Four sets of slides and two exposure durations were mixed up in each session. The slides exposed for $200 \mathrm{~ms}$ to the first group of 20 subjects were exposed for $50 \mathrm{~ms}$ to the second group. Those presented for $50 \mathrm{~ms}$ to the first group were presented for $200 \mathrm{~ms}$ to the second group. Consequently, all of the stimuli were presented for $50 \mathrm{~ms}$ and for $200 \mathrm{~ms}$ to either of two different groups of 20 subjects.

Sets a and $c$ of stimulus slides were randomly selected from Sets $A$ and $C$ used in Experiment 1. Each set consisted of 22 slides, two for each of 11 numerosities of dots, 4 to 14 . Sets $\mathrm{a}^{\prime}$ and $\mathrm{c}^{\prime}$ were new stimulus slides. Each of the two sets consisted of 30 slides, two for each of 15 numerosities of dots, 1 to 15 . The stimulus dots in Set $\mathrm{a}^{\prime}$ were random samples from the 100 dots in the $10 x$ 10 matrix shown in Fig. $4 \mathrm{a}^{\prime}$. The dots were distributed evenly in the whole area. In Set $c^{\prime}$, the stimulus dots were random samples from the 100 dots located in the four small $5 \times 5$ matrices shown in Fig. $4 \mathrm{c}^{\prime}$. The dots placed in four separate areas. They were easily seen as separate subgroups of dots as in Set c, but the pattern of dots in each subgroups was not so simple as in Set $c$, as indicated by an example shown in Fig. $4 \mathrm{e}$. The dots in

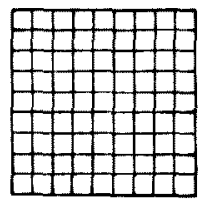

a'

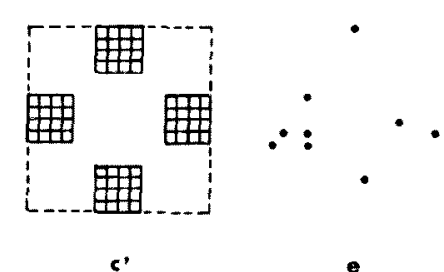

Fig. 4. ( $\left.a^{\prime}\right)$ and ( $c^{\prime}$ ) The matrices from whose vertices and points of intersection the positions of stimulus dots were sampled in the Sets $a^{\prime}$, and $c^{\prime}$. (e) An example of stimulus patterns used in the Set $c^{\prime}$ of Experiment 2. each subgroup consisted of random samples from a $5 \times 5$ matrix with a large variety of patterns, while those in Set $c$ had only a small variety. The diameter of each dots projected on the screen was $3.2 \mathrm{~cm}$ in Sets $\mathrm{a}$ and $\mathrm{c}$, and $2.6 \mathrm{~cm}$ in Sets $a^{\prime}$ and $c^{\prime}$. The center-to-center distance between the two nearest dots was $6.4 \mathrm{~cm}$ in Sets $a$ and $c, 7.2 \mathrm{~cm}$ in Set $a^{\prime}$ and $4.2 \mathrm{~cm}$ in Set $c^{\prime}$. The whole area of dot-pattern on the screen was $48 x$ $48 \mathrm{~cm}$ (5.5 to 12.5 degrees in visual angle for the furthest to the nearest subjects) in Sets a and $c$, and $65 \times 65 \mathrm{~cm}(7.5$ to 17 degrees in visual angle) in Sets $a^{\prime}$ and $c^{\prime}$.

The rest of the condition and procedure were the same as those in Experiment 1.

\section{Results and Discussion}

Because the number of repetitions was small, the obtained value for each numerosity of dots in each set was not stable enough to permit meaningful comparison among the three sets. Instead, the percentage of correct responses was pooled over the critical range of the number of dots from 8 to 11, for which the significant differences were found in Exp. 1, and the percentages of correct responses for the whole set were compared between stimulus sets and between the exposure durations, as shown in Table 1. The corresponding pooled percentage of correct responses in the other experiments are also shown in the same table.

The percentages of correct responses for Set $c$ were significantly greater than that for Set a, in both the exposure conditions, when the pooled data in the critical range of the numbers of dots ( 8 to 11) and those in the whole set were compared $\left(\chi^{2}=7.68\right.$ and $12.74, p<0.001$ in the $50 \mathrm{~ms}$ condition; $\chi^{2}=7.81, p<0.01$, and $\chi^{2}=4.78, p<$ 0.05 in the $200 \mathrm{~ms}$ condition). However, they were not significantly different between Sets $a^{\prime}$ and $c^{\prime}$, or significantly different in the opposite direction $\left(\chi^{2}=\right.$ $12.74, p<0.01$ for the critical range in the $50 \mathrm{~ms}$ condition). These results indi- 
Table 1

Percentages of correct responses in the critical range of the dot-numerosities and in the whole set

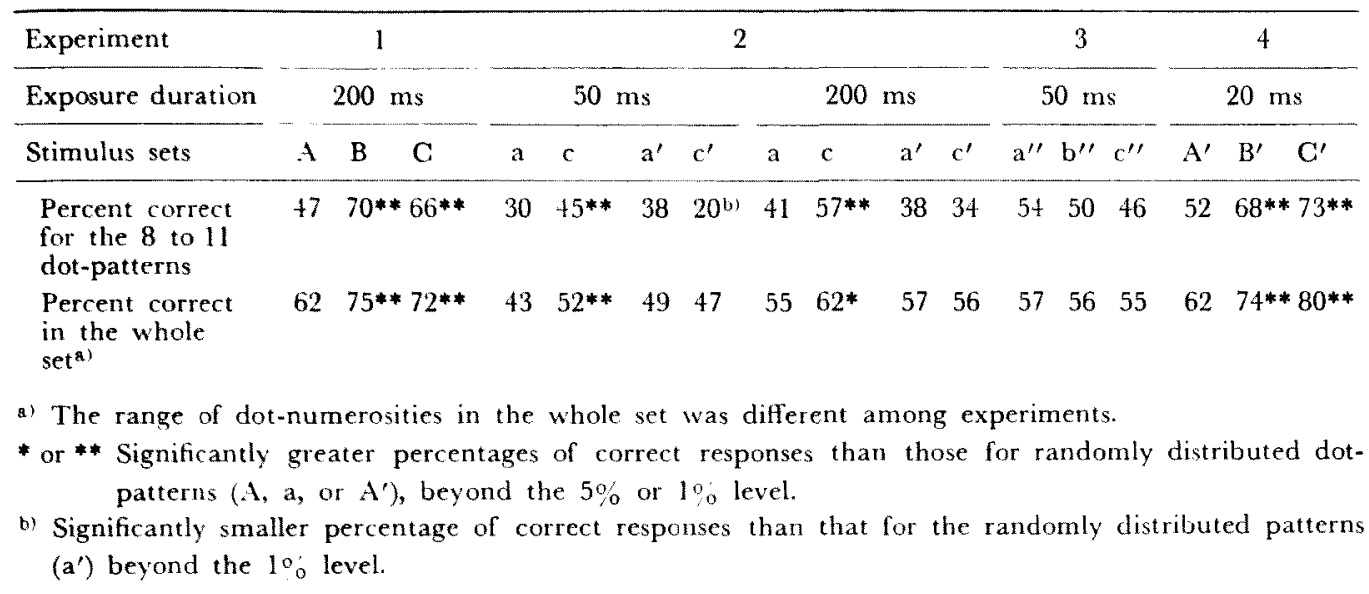

cate that better numerosity discrimination obtained in Sets B and C of Experiment $l$ and Set $c$ in the present experiment can be attributed to the simplicity of the patterns in each subgroup of dots. The spatial separation of subgroups does not improve numerosity discrimination, as shown by the results of Set $c^{\prime}$ in the present experiment. Another possible explanation of the superiority of Set $\mathrm{c}$ is that the number of dots in each subgroup was small (not greater than four), giving rise to possibility that each subgroup was subitized in a sense proposed by Kaufman, Lord, Reese, and Volkmann (1949). This possibility will be examined in the next experiment.

The percentages of correct responses for the whole set were significantly greater in the $200 \mathrm{~ms}$ exposure condition than in the $50 \mathrm{~ms}$ condition, for all sets, Sets a, $c$, $a^{\prime}$ and $c^{\prime}\left(\chi^{2}=10.47,9.37,6.64\right.$, and $10.00, p<0.01)$. This reconfirmed the finding of Hunter and Sigler (1940) that the prolongation of exposure duration beyond the critical duration of the temporal summation increases the limit of numerosity discrimination. The advantage of Set c over Set a was found in the $50 \mathrm{~ms}$ condition as well as in the $200 \mathrm{~ms}$ condition. This fact indicated that the advantage of Sets B and C over Set A in Exp. 1 was not specific to the exposure condition of $200 \mathrm{~ms}$.

\section{Experiment 3}

In this experiment, the numerosity discrimination was compared again with wellcontrolled stimulus presentation between randomly distributed dot-patterns and clustered dot-patterns under the condition that the dot-pattern in cach subgroup was not simple but the number of dots in it was always small (not greater than four).

\section{Method}

This experiment was conducted individually for four subjects. All of them were undergraduate students and had normal or corrected-to-normal visual acuity. Each participated in four sessions on different days.

A Takei three-channel tachistoscope was used. The viewing distance was $80 \mathrm{~cm}$, and $10 \mathrm{~mm}$ on the stimulus field subtended a visual angle of 0.72 degree. The luminance of the white surface of the stimulus cards in the tachistoscope was $15 \mathrm{~cd} / \mathrm{m}^{2}$.

Three sets of 132 stimulus patterns were used. Each set consisted of 12 different 
patterns for each of 11 numerosities of dots, from 4 to 14. Each stimulus pattern was made of black dots, $5 \mathrm{~mm}$ in diameter, on a white card. The dots in Sets a" were random samples from an $11 \times 11$ matrix of dots $(10 \times 10 \mathrm{~cm}$ in size, and $7 \times 7$ degrees in visual angle) shown in Fig. $5 \mathrm{a}^{\prime \prime}$. The dots in Set $b^{\prime \prime}$ were random samples from five $3 \times 3$ matrices shown in Fig. 5 b". The dots in Set c" were random samples from four $3 \times 3$ matrices shown in Fig. $5 c^{\prime \prime}$. In Sets $b^{\prime \prime}$ and c", four or less dots were sampled from each $3 \times 3$ matrix. This method produced a fairly complex dot-pattern in cach subgroup, though the variety of pattern was smaller than that in Set $c^{\prime}$ of Experiment 2. Different stimulus patterns were used in the four sessions, in different random order for each subject. In each session, 33 cards for each set were presented. Nine practice trials were conducted in the beginning of each scssion.

The subject was asked to fixate the center of the $21 \times 21 \mathrm{~cm}$ blank white field when the "Ready" signal was given and to report the perceived number of dots in the stimulus which was presented for $50 \mathrm{~ms}$, one second after the "Ready" signal. The white blank field was always presented between each stimulus. The subject was light-adapted to the luminance of the white field, during experiment.

\section{Results and Discussion}

Figure 6 shows the percentage of correct responses curves for the three stimulus

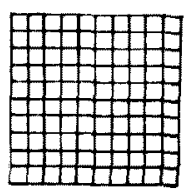

a"
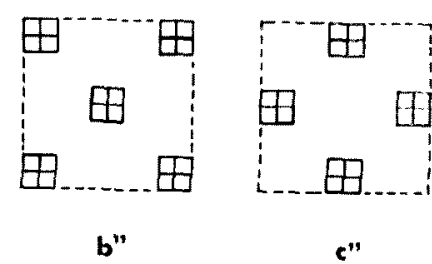

Fig. 5. The matrices from whose vertices and points of intersection the positions of stimulus dots were sampled in the Sets $a^{\prime \prime}, b^{\prime \prime}$ and $c^{\prime \prime}$ of Experiment 3 . sets as a function of the presented number of dots. Three curves do not show any systematic difference among the three sets. The total percentages of correct responses for the critical range of the number of dots, 8 to 11 , and for the whole sets were nearly the same for the three sets as shown in Table 1. No statistical difference was found among them. The span-of-attention values calculated from the percentage of correct responses curves were $9.4,9.1$, and 9.1 for Sets $a^{\prime \prime}, b^{\prime \prime}$, and $c^{\prime \prime}$, respectively. These values are very similar to one another. No advantage of Sets $b^{\prime \prime}$ and $c^{\prime \prime}$ (clustered dots with complex and irregular patterns in subgroups) over Set a" (randomly distributed dots) was found. These results, together with the results of Experiments 1 and 2, indicated that the simplicity and regularity of patterns in subgroups are the critical factors for improving the numerosity discrimination in such patterns as Sets B and $\mathrm{C}$ of Experiment 1 and Set $c$ in Experiment 2. The response to simple and regular, or "canonical " patterns is more accurate than to randomly generated patterns, as indicated by Mandler and Shebo (1982). This finding is also in line with those reported by Atkinson, Francis, and Campbell (1976) and Fujii (1967), who both used simple and regular linear arrays of equally spaced dots or lines as spatially separated subgroups of

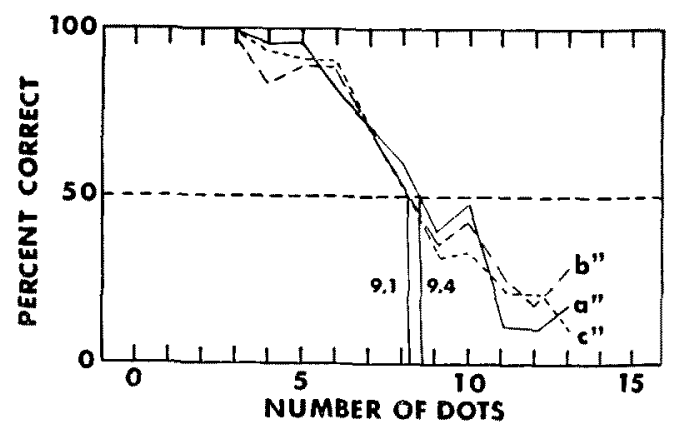

Fig. 6. Percentage of correct responses as a function of the number of dots for the Sets $a^{\prime \prime}, b^{\prime \prime}$, and $c$ " of Experiment 3. 
the stimulus objects and found a similar effect of grouping on numerosity discrimination.

So, how does simplicity and regularity of patterns in subgroups bring better numerosity discrimination? This problem will be examined using reaction times, which have proven to be a useful measure for analyzing the process of numerosity discrimination (Oyama et al., 1981).

\section{Experiment 4}

In this experiment, an attempt was made to analyse the numerosity discrimination processes for stimulus patterns, which were subdivided into subgroups with simple and regular forms, using reaction time as the measure. Another purpose of this experiment is reconfirmation of the results of Experiment 1 in a well-controlled condition.

\section{Method}

This experiment was also conducted individually for 13 subjects. All were undergraduate students and had normal or corrected-normal visual acuity. Each participated in both two sessions.

A Ralph Gerbrands four-channel tachistoscope was used, but only two channels were utilized in this experiment. The viewing distance was $80 \mathrm{~cm}$. The stimuli were light dot-patterns illuminated from back light boxes attached to the channels. The luminance of luminous dots was $25 \mathrm{~cd} / \mathrm{m}^{2}$.

Three sets of 60 test stimuli were prepared. They were black cards containing small circular holes $(5 \mathrm{~mm}$, or 0.36 degree, in diameter). One to fifteen holes were included in a test stimulus. These holes in Set $\mathrm{A}^{\prime}$ of the test stimuli were placed in positions randomly sampled from an $8 \times 8$ matrix $(7 \times 7 \mathrm{~cm}$ in size, $5 \times 5$ degrees in visual angle), as in the black dots in Set A of Experiment 1. Those in Sets $B^{\prime}$ and $\mathrm{C}^{\prime}$ of the test stimuli were placed in positions sampled with the same principle as in Sets B and $C$ of Experiment 1, from four or five separate parts of the same matrix. A total of 180 test stimuli, four different patterns for each of 15 numerosities of dots, 1 to 15 , in each of three stimulus sets, were prepared. In each session, a half of them, two patterns for 15 numerosities in three sets, were mixed up and presented in a different random order for each subject, after five practice trials with different stimuli of the same type.

In each trial, after the experimenter's "Ready" signal, a small red dot $(3 \mathrm{~mm}$ in diameter) was presented at the center of the dark stimulus field for $400 \mathrm{~ms}$, which was followed by an one-second dark interval, and then by a test stimulus for $20 \mathrm{~ms}$. The subject was asked to fixate the small red dot when it was presented, and to report the perceived number of the test dots as quickly as possible. The reacton time was measured by a voice key. The subject was adapted to the room light during the experiment.

\section{Results and Discussion}

Three measures, the percentage of correct responses, the mean reported number of dots, and the mean reaction time were compared among the three sets of stimulus for each numerosity of stimulus dots.

Figure 7 shows the percentage of correct responses for the three stimulus sets

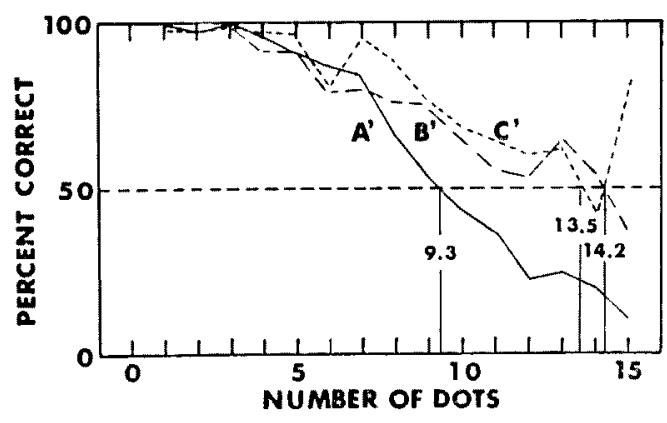

Fig. 7. Percentage of correct responses as a function of the number of dots for the Sets $A^{\prime}, B^{\prime}$, and $\mathrm{C}^{\prime}$ of Experiment 4. 
as a function of the number of stimulus dots. The solid, broken, and dotted lines represent the results of Sets $A^{\prime}, B^{\prime}$, and $C^{\prime}$, respectively. The general trend was the same as that found in Experiment 1. The percentage of correct responses was clearly higher in Sets $B^{\prime}$ and $C^{\prime}$ than in Sets $A^{\prime}$, especially when the number of dots was greater than 7. Chi-square tests showed that the percentage of correct responses was significantly greater in Set $\mathrm{B}^{\prime}$ than in Set $\mathrm{A}^{\prime}$ for the stimulus patterns with 9 , $10,12,13,14$, and 15 dots $\chi^{2}=5.07$, and $4.66, p<0.05$ for 9 and 10 dots; $\chi^{2}=11.85$, $17.27,13.43$, and $9.43, p<0.01$ for 12 to 15 dots), and significantly greater in Set $\mathrm{C}^{\prime}$ than in Set $\mathrm{A}^{\prime}$ for the stimulus pattern with $8,9,10,12,13,14$, and 15 dots $\left(\chi^{2}=6.37,5.07,6.56\right.$, and $5.56, p<0.05$ for $8,9,10$, and 14 dots; $\chi^{2}=15.97,15.75$, and $55.89, p<0.01$ for 12,13 , and 15 dots). The high percentage of correct responses obtained for 15-dot-patterns in Set $C^{\prime}$ might reflect the fact that the stimulus patterns were lacking only one dot to produce a regular four-square pattern and the subjects only needed to find this missing dot to response correctly.

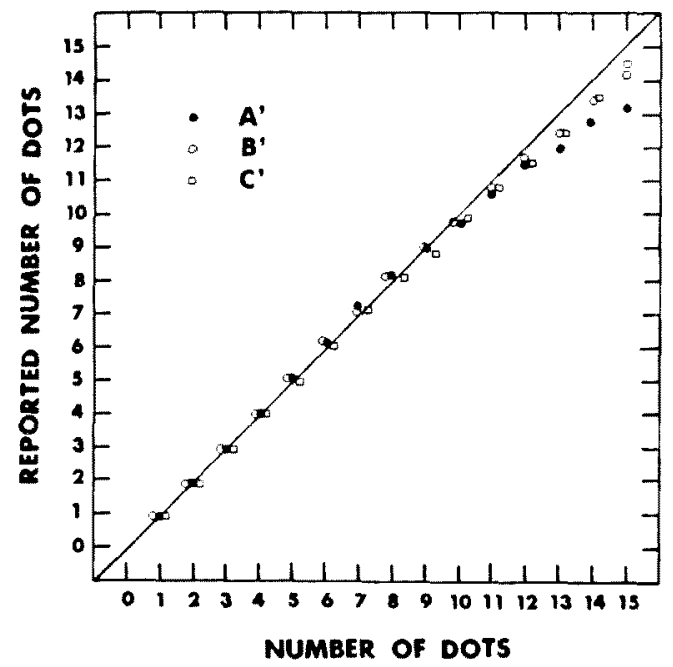

Fig. 8. The mean reported number of dots as a function of the actual number of stimulus dots for the Sets $A^{\prime}, B^{\prime}$, and $C^{\prime}$ of Experiment 4 .
The span-of-attention values calculated from the percentage of correct responses curves were 9.3 in Set $\mathrm{A}^{\prime}, 14.2$ in Set B', and 13.5 in Set $C^{\prime}$. These values were definitely greater in Sets $B^{\prime}$ and $C^{\prime}$ than in Set $\mathrm{A}^{\prime}$, as in Experiment 1 .

Figure 8 shows the mean reported number of dots as a function of the actual number of stimulus dots. The three symbols represent the three stimulus sets. The general trend was similar in all three sets and to the results obtained by Oyama et al. (1981) in their no-mask condition. The mean reported numbers of dots was nearly the same as the actual numbers of dots when the stimulus pattern included fewer than 10 dots, but there was a tendency to underestimate the number of dots for the stimulus patterns with 10 or more dots. It is especially interesting that this trend toward underestimation was smaller in Sets $B^{\prime}$ and $\mathrm{C}^{\prime}$ than in Set $\mathrm{A}^{\prime}$. The results of $t$-tests indicated that the reported numbers of dots were significantly greater in Sets $B^{\prime}$ and $\mathrm{C}^{\prime}$ than in Set $\mathrm{A}^{\prime}$ for 13 to 15 stimulus dots $(t=2.13$ and $2.11, p<0.05$ for 13 dots; 3.06 and $3.12, p<0.01$ for 14 dots;

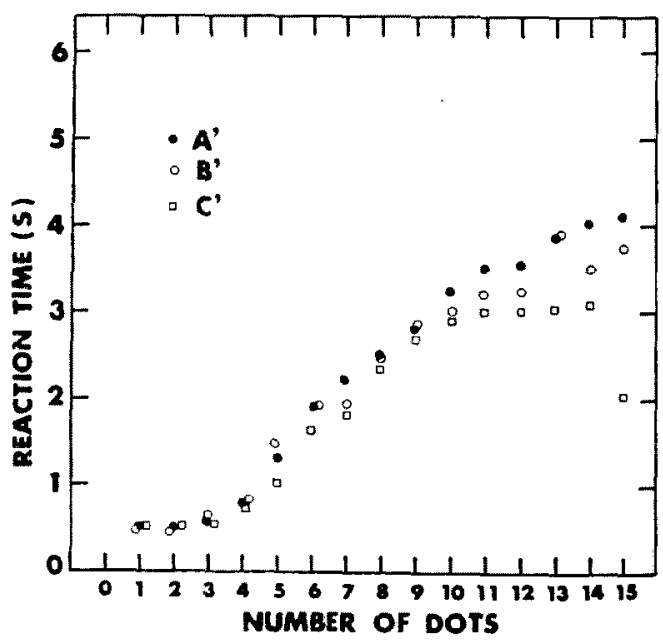

Fig. 9. The mean reaction time as a function of stimulus dots for the Sets $\mathrm{A}^{\prime}, \mathrm{B}^{\prime}$, and $\mathrm{C}^{\prime}$ of $\mathrm{Ex}$ periment 4. 
4.63 and $6.14, p<0.01$ for 15 dots). This result indicates that the mean reported numbers of dots were closer to the actual numbers of dots in Sets $B^{\prime}$ and $C^{\prime}$ than in Set $A^{\prime}$.

Figure 9 shows the mean reaction time as a function of the number of stimulus dots. In general, the mean reaction time increased linearly at a low rate for stimuli with 1 to 4 dots and then increased linearly again at a higher rate for stimuli with more than 4 dots. This finding was the same as that found by Oyama et al. (1981), any difference being that, in the present experiment, the rate of increasc becomes smaller when the number of dots increased beyond 10 , especially in Sets $B^{\prime}$ and $C^{\prime}$. The results of $t$-test indicated that the reaction time was significantly smaller in Set $B^{\prime}$ than in Set $A^{\prime}$ for 7-dot patterns $(t=2.07, p<0.05)$, and smaller in Set $C^{\prime}$ than in Set $A^{\prime}$ for 5-, 6-, 7-, 11-, 12-, 13-, 14-, and 15-dot patterns $(t=2.44$ and $2.84, p<0.05$ for 6 and 7 dots; $t=3.14,2.88,3.04,3.57,5.11$, and $9.27, p<0.01$ for $5,11,12,13,14$, and 15 dots.) In fitting linear regression lines to the reaction times to 1 to 4 dots and those to 4 to 15 dots, the following regression coefficients, or the rates of increase, were obtained. Low rates of increase, 89, 105, and $72 \mathrm{~ms}$ per dot, for 1 to 4 dots in Sets $\mathrm{A}^{\prime}, \mathrm{B}^{\prime}$ and $\mathrm{C}^{\prime}$ which might reflect the subitizing process, named by Kaufman et al. (1949), and high rates of increase, 294, 264, and $178 \mathrm{~ms}$ per dot for 4 to 15 dots in Sets $A^{\prime}, B^{\prime}$, and $\mathrm{C}^{\prime}$, which may correspond to the counting process (Klahr, 1973; Oyama et al., 1981).

It should be noted that the fitness of a linear line to the reaction times for 4 to 15 dots was poor in Set $C^{\prime}$, reflecting the stop of increase of the reaction time for more than 10 dots in Set $\mathrm{C}^{\prime}$. This fact suggested that the subject used a different strategy from that used in Set $A^{\prime}$, in this set, to count 11 to 15 dots. The subject might subitize the number of dots in each subgroup and add the result to the running total, as in one of Klahr's (1973) models. In this subitizing-and-addition strategy, the reaction time depends largely on the number of subgroups and partly on the numbers of dots in subgroups, because the time needed for subitizing increases only slightly with the number of dots, as compared with the time needed for addition. This subitizing-and-addition model fits the reaction times for more than 10 dots in Set C'. A similar but weaker tendency can also be observed in Set $\mathbf{B}^{\prime}$. These results on reaction times suggested that better numerosity discrimination was made in Sets $B^{\prime}$ and $C^{\prime}$, in which stimulus patterns tended to be seen as four or five subgroups of dots, each having a simple and regualr form, resulting in the subjects' faster processing of seen dots. By faster processing, greater numbers of dots can be processed before the disappearance of the visual image, or visual short-term memory, of the stimulus pattern, which was estimated to continue for a little over two seconds in the previous investigation (Oyama et al., 1981).

The results of Experiments 2 and 3 showed that better numerosity discrimination for subdivided patterns is limited to simple and regular subgroup-patterns. In spite of the fact that single irregular patterns of less than 5 dots can be subitized as shown in the results of Set $A^{\prime}$ of this experiment, the subitizing-and-addition strategy did not seem to be used when four or five irregular patterns were contained simultaneously in a stimulus. Thus, the subitizing of irregular pattern would be limited to the condition in which such a pattern is presented singly, although the subitizing of regular and simple patterns is possible even when four or five of such patterns are presented in parallel.

\section{Conclusions}

1) Numerosity discrimination improves 
when the stimulus dots consist of spatially separated subgroups, as compared with those distributed in the whole area with equal probability (Experiments 1 and 4).

2) However, this effect is only limited to the condition that dot-patterns in subgroups are simple and regular. Numerosity discrimination is not improved when dot-patterns in spatially separated subgroups are complex and irregular (Experiments 2 and 3 ). These results suggest that, contrary to the expectation, perceptual grouping is not necessarily accompanied by the improvement of numerosity discrimination. Negative results of Atkinson, Francis, and Campbell (1976) and of the author's unpublished study on the effect of grouping induced by colorsimilarity also support this view.

3) Reaction times for numerosity discrimination is shorter when the stimulus patterns consist of simple-form subgroups of dots than random-dot patterns, especially for more than 10 dots (Experiment 4).

4) The improved numerosity discrimination in these stimulus patterns is attributed to more rapid perceptual processing, by means of a strategy in which stimulus-dots in each subgroup are subitized and then summed up together. This strategy makes it possible for the subject to process more dots in the limited time of the persistence of the visual image or visual short-term memory of the stimulus pattern.

\section{References}

Atkinson, J., Campbell, F. W., \& Francis, M. R. 1976 The magic number $4 \pm 0$ : A new look at visual numerosity judgements. Perception, 5, 327334.

Atkinson, J., Francis, M. R., \& Campbell, F. W. 1976 The dependence of the visual numerosity limit on orientation, colour, and grouping in the stimulus, Perception, 5, 335-342.

Fujii, M. 1967 Experimental investigations on the instantaneous judgment of the number of objects (II). Japanese Journal of Psychology, 38, 8393. (In Japanese with English summary)

Hamilton, W. 1859 Lectures on metaphysics and logic. Vol. 1. Edinburgh: Blackwood.

Hunter, W. S., \& Sigler, M. 1940 The span of visual discrimination as a function of time and intensity of stimulation. Journal of Experimental Psychology, 26, 160-179.

Jevons, W.S. 1871 The power of numerical discrimination. Nature, 3, 281-282.

Kaufman, E. L., Lord, N. W., Reese, T. W., \& Volkmann, J. 1949 The discrimination of visual number. American Journal of Psychology, 62, 498-525.

Klahr, D. 1973 Quantification process. In W. G. Chase (Ed.), Visual information processing. New York: Academic Press. Pp. 3-34.

Mandler, G., \& Shebo, B.J. 1982 Subitizing: An analysis of its component processes. Journal of Experimental Psychology: General, 111, 1-22.

Miller, G. A. 1956 The magical number seven, plus or minus two: Some limits on our capacity for processing information. Psychological Review, 63, 81-97.

Oyama, T., Kikuchi, T., \& Ichihara, S. 1981 Span of attention, backward masking, and reaction time. Perception and Psychophysics, 29, 106112.

Wertheimer, M. 1923 Untersuchungen zur Lehre von der Gestalt, II. Psychologische Forschung, 4, $30 \mathrm{~L}-350$.

Woodworth, R. S., \& Schlosberg, H. 1954 Experimental Psychology. (Re. ed.) New York: Holt, Rinehart and Winston.

(Received May 31, 1985; accepted Jan. 18, 1986) 